\title{
Prevalence of Diabetic Keto Acidosis and Associated Factors among Newly Diagnosed Patients with Type One Diabetic Mellitus at Dilla University Referral Hospital, September 9th/2017 - May 30th/2019: South Ethiopia; Crossectional Study
}

\author{
Alem Eskeziya $^{1^{*}}$, Zeleke Girma ${ }^{2}$, Baharu Mandefreo $^{3}$ and Amare Haftu ${ }^{3}$ \\ ${ }^{1}$ Department of Psychiatry, College of Health Science, Dilla University, Dilla, Ethiopia \\ ${ }^{2}$ Department of Public Health, College of Health Science, Dilla University, Dilla, Ethiopia \\ ${ }^{3}$ Department of Internal Medicine, School of Medicine, Dilla University, Dilla, Ethiopia
}

\begin{abstract}
Background: Diabetic Ketoacidosis (DKA) is one of the most serious acute complications of Diabetes Mellitus (DM) and the leading cause of morbidity and mortality in young adults with type 1 diabetes. The mortality rate for DKA ranges from 2 to $5 \%$ in developed countries and 6 to $24 \%$ in developing countries. Even though many DKA patients were seen in emergency unit of Ethiopian hospitals and health centers, little is known about precipitating factors and clinical-laboratory features of DKA among Ethiopian patients and information is scant to promote better health service to prevent mortality due to DKA. So the aim of this study is to assess the prevalence and associated risk factors of DKA in newly diagnosed Type 1 Diabetic patients in Dilla University Referral Hospital (DURH) from September 9 $/ 2017-$ May $30^{\text {th }} / 2019$.
\end{abstract}

Method: Quantitative institutional based study was conducted among 421 newly diagnosed type one diabetic patients. Data was double entered from the paper-based abstraction sheet into Epi info version 7 and exported to SPSS version 20 for analysis. A descriptive analysis was performed. Bivariate and multivariate logistic analysis was done to identify factors associated with the magnitude of Keto acidosis. Variables with p-value less than 0.05 were declared as having significant association between factors and dependent variable.

Result: The magnitude of Diabetic Keto Acidosis (DKA) in newly diagnosed patients with type one diabetic Mellitus (T1DM) was found to be $38 \%$. The significant predictors of Diabetic Keto Acidosis (DKA) among newly diagnosed patients with type one diabetic Mellitus (T1DM) were young age of the adult, family history of diabetes and infection prior to onset of Diabetic Keto Acidosis (DKA).

Conclusion: The overall magnitude of Diabetic Keto Acidosis (DKA) in adults with newly diagnosed type 1 diabetes is high. In particular, adults between 18-25 years of age have a high risk of Diabetic Keto Acidosis (DKA) at onset of diabetes mellitus. Young Age, first degree relatives with Diabetic Mellitus (DM) and infection prior to Diabetic Keto Acidosis (DKA) are found to be the significant explanatory variable of Diabetic Keto Acidosis (DKA) in primary onset of T1DM.

\section{Keywords}

Diabetic keto acidosis, Newly diagnosed patients, Diabetic mellitus

\section{List of Abbreviation}

CDC: Centre for Disease Control; ETB: Ethiopian Birr; DM: Diabetes Mellitus; DKA: Diabetic Keto Acidosis; DURH: Dilla University Referral Hospital; HHS: Hyperosmolar Hyperglycemic State; FMOH: Federal Ministry of Health; T1: Type One; WHO: World Health Organization

\section{Introduction}

A normal blood sugar level for a person who has not been diagnosed with diabetes should be $80-99 \mathrm{mg} / \mathrm{dL}$ (milligrams per deciliter) when they have not eaten for more than two hours. Diabetes is a disease that affects blood sugar management in the human body, it means the amount of glucose or sugar in your blood typically remains too high and exceeds blood sugar levels that are considered normal. This occurs

Copyright: (C) 2020 Eskeziya A, et al. This is an open-access article distributed under the terms of the Creative Commons Attribution License, which permits unrestricted use, distribution, and reproduction in any medium, provided the original author and source are credited. 
Citation: Eskeziya A, Girma Z, Mandefreo B, et al. (2020) Prevalence of Diabetic Keto Acidosis and Associated Factors among Newly Diagnosed Patients with Type One Diabetic Mellitus at Dilla University Referral Hospital, September 9th/2017 - May 30th/2019: South Ethiopia; Crossectional Study. J Healthcare 3(1):33-38

when there is a problem with the insulin response in the body [1].

Diabetes mellitus (DM) is the commonest metabolic diseases all over the world. The worldwide prevalence of DM has risen dramatically over the past two decades, from an estimated 30 million cases in 1985 to 177 million in 2000. Based on current trends, Above 360 million individuals will have diabetes by the year 2030. In the United States, the center for Disease Control and Prevention (CDC) estimated that 20.8 million persons, or $7 \%$ of the population, had diabetes in 2005 and around $30 \%$ of individuals with diabetes were undiagnosed. In Africa 7 million individual had diabetes in 2000 and this number increase to 18.2 million on 2030. Worldwide estimates project that in 2030 the greatest number of individuals with diabetes will be $45-64$ years of age [1,2]. World health organization (WHO) estimates the number of cases of diabetics in Ethiopia to be about 800,000 in 2000 and by this protestation it would increase to about 1.8 million by the year 2030 [2]. About 3\% of admission to medical wards in Ethiopian hospitals is due to diabetes mellitus [3]. Diabetes mellitus leads to acute and chronic complications.

The acute complications include Diabetic keto Acidosis (DKA), hyperosmolar hyperglycemic state (HHS), and hypoglycemia during treatment. The chronic complications are neuropathy, nephropathy, retinopathy, ischemic heart disease, myocardial infarction, stroke, peripheral arterial disease, impotence and so on [4]. Diagnosis of diabetic ketoacidosis is based on clinical features, serum sugar $>12 \mathrm{mmol} /$ with ketonuria, bicarbonate and base deficit. The mean age was 22.5 years with a male to female ratio of 1.4:1 [5].

Diabetic Keto Acidosis (DKA) is the most common hyperglycemic emergency in patients with diabetes mellitus. DKA most often occurs in patients with type 1 diabetes mellitus. Worldwide, infection is the most common precipitating cause for DKA, occurring in $30-50 \%$ of cases. Urinary tract infection and pneumonia account for the majority of infection site. Other precipitating causes are like surgery, trauma, myocardial ischemia, pancreatitis, psychological stress, and non-compliance with insulin therapy contribute to DKA [6].

In the study carried in 2004 out at Grand Memorial Hospital Atlanta, Georgia, to identify the causes of diabetic ketoacidosis (DKA), there were 56 episodes of DKA, and 75\% of the episodes were in patients with known diabetes [7]. A study conducted in King Abdul-Aziz University Hospital, Jeddah, Kingdom of Saudi Arabia revealed that Poor compliance (54.4\%) and infection (28\%) were the most common precipitating factors being responsible for DKA cases [5]. A retrospective cohort study conducted in Soroka University Medical Center, Beer Sheva showed that the most frequent precipitating factors were related to insulin therapy and infections [8].

A 10 years (1998-2008) retrospective study conducted in Ilorin teaching hospital, Nigeria $(n=225)$ shows that 172 patients admitted because of DKA precipitated by septicemia and 36 DKA patients precipitated by meningitis [9]. In the study carried out in urban hospital of Nigeria A total of 94 subjects who presented With Diabetes ketoacidosis (DKA) DKA occurred in all subjects with type 1 DM [10].
In Literature review of relevant published literature from both Africa and the rest of the world, the main causes or precipitants of DKA in patients in sub-Saharan Africa were newly diagnosed diabetes, missed insulin doses and infections $[11,12]$.

In study conducted in Ethiopia the clinical feature of 44 episodes of diabetic ketoacidosis in 34 patients with type 1 diabetes mellitus in Ethiopian, the mortality rate was only $9.1 \%$, even though serum potassium, bicarbonate and $\mathrm{pH}$ could not be measured. Although 3 patients from 44 were new diabetics, and the cause was not found in 7 , most cases resulted from omission of insulin (43\%) or recurrent illness (28\%). Socioeconomic factors, particularly the cost and unreliability of insulin supplies, are major obstacles to the control of diabetes and the prevention of ketoacidosis among patients with type 1 diabetes mellitus in Ethiopia [13]. A clinical survey conducted in Yekatit 12 hospital, between 2002 and 2004 ( $n=$ 44 patients) only 3 patients were previously undiagnosed diabetes. A misguided attempt at treatment with oral hypoglycemic agents aggravated 4 episodes, and intermittent illness was responsible for 15 patients, but no precipitating cause was identified for 7 patients. In 19 patients of 44 episodes $(43 \%)$ the most important precipitating factor was omission of insulin. The reason for omission of insulin was inability to obtain it either because of the patient couldn't afford it and failed to acquire paper for free health care or because the health center or hospital have no insulin [13].

The mortality rate for DKA ranges from 2 to 5 percent in developed countries and 6 to 24 percent in developing countries. If it misdiagnosed or mistreated it is $100 \%$ fatal and in places like Ethiopia where medical services are less developed, the risk of dying from DKA is greater [14-16]. In developing countries like Ethiopia, due to widespread poverty of individuals the healthcare systems are scarce and the few available centers are unable to adequately maintain a reliable system of insulin supply and exhaustively investigate their hospitalized patients. Consequently, there is little guarantee of successful outcomes. Poor people may also have sub-optimal nutrition, caused or worsened by diabetes, specially, at first presentation to hospital. Intensive insulin therapy in such individuals mimics re-feeding syndrome, an acute anabolic state whose outcome may be unfavorable during the period of treatment of diabetic ketoacidosis [17].

DKA in the Ethiopian care setup has not been well documented [2]. Many patients with diabetes keep dying from DKA every year [17]. With the current trend of transition from

*Corresponding author: Alem Eskeziya, Department of Psychiatry, College of Health Science, Dilla University, Dilla, Ethiopia

Accepted: August 29, 2020

Published online: August 31, 2020

Citation: Eskeziya A, Girma Z, Mandefreo B, et al. (2020) Prevalence of Diabetic Keto Acidosis and Associated Factors among Newly Diagnosed Patients with Type One Diabetic Mellitus at Dilla University Referral Hospital, September 9th/2017 - May 30th/2019: South Ethiopia; Crossectional Study. J Healthcare 3(1):33-38 
Citation: Eskeziya A, Girma Z, Mandefreo B, et al. (2020) Prevalence of Diabetic Keto Acidosis and Associated Factors among Newly Diagnosed Patients with Type One Diabetic Mellitus at Dilla University Referral Hospital, September 9th/2017 - May 30th/2019: South Ethiopia; Crossectional Study. J Healthcare 3(1):33-38

communicable to non-communicable disease, it is projected that the latter will equal or even exceed the former in developing nations, thus culminating in double burden. For large areas of the World, particularly in Africa and South East Asia, there are few data or none at all, on the frequency of DKA in adults.

The magnitude of DKA in adults in Africa from few pocket studies is much higher than the developed countries. In Ethiopia like other African countries the magnitude and risk factors of DKA in newly diagnosed DM adults is not well studied. Without the knowledge of the magnitude and associated risk factors of DKA in adults the preventable associated risk factors Even though many DKA patients was seen in emergency unit of Ethiopian hospitals and health facilities, Little is known or very little study is done about precipitating factors and clinical-laboratory features of DKA among Ethiopian patients and information is scant to promote better health service to prevent mortality due to DKA. Knowledge about precipitating factors of DKA will give insight about uniform approach of diagnosis. The information gained from this study will be step towards for supporting and promoting early management, planning and prevention of DKA. The finding of this study may be used as a base line data for those who are interested in carrying out further study.

\section{Methods}

This study was conducted in Dilla University Referral Hospital (DURH), Dilla. It is located $360 \mathrm{~km}$ from the capital city, (Addis Ababa), and $90 \mathrm{~km}$ from the Hawasa (capital city of south region). Dilla university referral hospital situated in the Dilla town of Gedeo Zone and serves a population of over 550,000 who are mainly (90\%) rural. It is the only referral hospital with a total of 165 beds that provides surgical, gynecologic/obstetric, medical, and pediatric wards in the Gedeo Zone. It acts as a referral center for the surrounding primary health care unit. The main diagnostic modalities in this hospital are routine laboratory investigation, radiological and pathological service. It has physicians within certain specialties such as surgery, orthopedics, medicine, pediatrics, dermatology gynecology, radiology, dental doctor and anesthesia on duty on 24 hour bases. An institution based cross sectional study was conducted from September $9^{\text {th }} / 2017-M a y 30^{\text {th }} / 2019$ on 421 randomly selected newly diagnosed patients with type 1 diabetes mellitusthat were admitted in medical emergency in DURH from September 2017 till $1^{\text {st }}$ May 2019 G.C. Sample size was calculated using single population proportion with the following assumptions.

$$
n=\frac{(z 1-\alpha / 2) 2 \times p(1-p)}{d 2}
$$

Where; $n=$ Minimum sample size for a statistically significant survey, $\mathrm{Z}=$ Normal deviant at the portion of $95 \%$ confidence interval two tailed test $=1.96, \mathrm{P}=58 \%$ (similar previous study), $q=1-p, d=$ allowable margin $=5 \%$. Accordingly, Minimum sample size $(n)=421$.

The sample size was also adjusted to compensate for non-response rate of $10 \%$. Hence, total sample sizes of 421 of newly diagnosed Type one Diabetic patients were used in the study. Systematic random sampling technique was used. With $\mathrm{k}=6$ List of all DM /DKA patients in newly diagnosed T1DM from registration log book will be used as sampling frame. Before data collection ethical clearance per protocol unique number: 007/17-13 was secured from Institutional Review Board (IRB) of Dilla university on March $3^{\text {rd }} / 2019$. Permission letter was taken from medical director of Dilla university hospital. Verbal consent from each study subject was asked and secured after detail explanation about the main purpose of the study, the confidentiality of the information was assured by anonymous of the study subjects in the questioners and large effort was made to maintain privacy of the respondents during the interview After explaining the purpose of the study in detail to the study participants and informing them that they have a full right to cooperated and participated and not to participate or even to interrupt and leave the interview if they feel discomfort. Maximum effort was made to maintain privacy and confidentiality during data collection. Data was collected by clinical nurses using prepared checklist, from registration log book. And chart of the patients were revised. EPI info.3.1 and SPSS version 20 was used for data entry, editing and analysis, respectively. The collected data were double entered using EPI data software version 3.1. Then it was exported to SPSS version 20.0 for data processing and analysis. The following variables were measured as follows during analysis: (a) Diabetic Ketoacidosis in newly diagnosed adults:- Adults who have blood glucose level $\geq 250 \mathrm{mg} / \mathrm{dl}$, Ketonuria and ketonemia and diagnosed being Diabetes Mellitus patient for the first time; (b) Adult Age :- > 18-years-old; (c) First degree relatives with DM:- Child's mother, father or sibling with DM; (d) Preceding infection:- Any infection 1-2 weeks before the onset of DKA; (e) Preceding sign and symptoms of DM/DKA:$A$ adult who had polyuria, polydepsia and weight loss 1-2 week prior to DKA; (f) Knowledge of the sign and symptoms of DM/DKA:- Parents who know the three poly symptoms (polyuria, polydepsia and polyphagia) and weight loss were considered knowledgeable. Descriptive tests like proportions mean and standard deviations and analytic tests like bivariate and multivariate logistic regression analysis were computed. Hosmer-Lemeshow goodness-of-fit was used to test model fitness.Finally, Variables with $p$-value less than 0.05 were declared as having significant association between factors and dependent variable.

\section{Results}

\section{Socio-economic and demographic characteris- tics of respondents}

A total of 421 charts of newly diagnosed patients with type 1 diabetes mellitus between September 2017 and May 2019 were reviewed of 421 adults 89 (21.1\%) was orthodox Christian, 90 (21.4\%) Muslims and 185 (43.9\%) were protestants. Regarding educational levels of the patients, 64 (15.2\%) can read and write, 81 (19.2\%) are on primary school, 254 (60.3\%) are high school level and 22 (5.2\%) are above highschool (Table 1).

\section{Clinical characteristics of respondents}

Among the 421 newly diagnosed patients with type 1 
Citation: Eskeziya A, Girma Z, Mandefreo B, et al. (2020) Prevalence of Diabetic Keto Acidosis and Associated Factors among Newly Diagnosed Patients with Type One Diabetic Mellitus at Dilla University Referral Hospital, September 9th/2017 - May 30th/2019: South Ethiopia; Crossectional Study. J Healthcare 3(1):33-38

Table 1: Socio-demographic characteristics newly diagnosed type1 DM adults who developed DKA from September 2017 to May 2019 G.C in DURH.

\begin{tabular}{|c|c|c|c|}
\hline \multicolumn{2}{|l|}{ Variables } & \multirow{2}{*}{$\begin{array}{l}\text { Frequency } \\
230 \\
191\end{array}$} & \multirow{2}{*}{\begin{tabular}{|l} 
Percent (\%) \\
54.6 \\
45.4
\end{tabular}} \\
\hline Sex & Male Female & & \\
\hline Religion & $\begin{array}{l}\text { Orthodox } \\
\text { Muslim } \\
\text { Protestant } \\
\text { Catholic }\end{array}$ & $\begin{array}{l}89 \\
90 \\
185 \\
57\end{array}$ & $\begin{array}{l}21.1 \\
21.4 \\
43.9 \\
13.5\end{array}$ \\
\hline Ethnicity & $\begin{array}{l}\text { Gedeo } \\
\text { Wolaita } \\
\text { Silte } \\
\text { Amhara } \\
\text { Oromia }\end{array}$ & $\begin{array}{l}63 \\
61 \\
133 \\
15 \\
149\end{array}$ & $\begin{array}{l}15.0 \\
14.5 \\
31.6 \\
3.6 \\
35.4\end{array}$ \\
\hline $\begin{array}{l}\text { Educational } \\
\text { level }\end{array}$ & $\begin{array}{l}\text { Read and write } \\
1-8 \\
\text { high school } \\
\text { complete } \\
\text { above high school }\end{array}$ & $\begin{array}{l}64 \\
81 \\
254 \\
22\end{array}$ & $\begin{array}{l}15.2 \\
19.2 \\
60.3 \\
5.2\end{array}$ \\
\hline Monthly income & $\begin{array}{l}<1000 \text { ETB } \\
1000-5000 \text { ETB } \\
>5000 \text { ETB }\end{array}$ & $\begin{array}{l}8 \\
314 \\
99\end{array}$ & $\begin{array}{l}1.9 \\
74.6 \\
23.5\end{array}$ \\
\hline
\end{tabular}

diabetes mellitus from September 2017 to May 2019, 160 (38.0\%) were found to have DKA and type 1 DM at the same time (they are newly diagnosed), and 261 (62.0\%) were only diagnosed with type 1 DM but without DKA. In this study the frequently reported presenting symptoms of DKA were 117 (27.8\%) present with altered mentation, 127 (30.2\%) with dry mouth which is the sign of dehydration and vomiting and only $4(1 \%)$ of adults present fast breathing (Table 2).

\section{Factors associated with diabetic keto acidosis (DKA)}

In multivariate analysis age of the adult, family history DM, and preceding infection found to have significant association with DKA at primary diagnosis of DM. Although males presented more frequently (54.6\%) with DKA than did females $(45.4 \%)$, the difference was not significant ( $p=0.98$ ). Clients between $18-25$ years-old have significant association with developing DKA in AOR of $3(1,10)$ with $p$ value of 0.005 (Table 3).

\section{Discussion}

In the present study, slightly above one third of adults manifested with DKA at first diagnosis of diabetes. This is not surprising as a similar low magnitude has been reported in a previous study in Italy [15]. The same is true of reports from Iran (24\%) [13], Sweden (14\%), Canada (18.6\%), Finland (22\%) and Hungary (23\%) [8]. And lower than the findings of previous studies done in UAE (80\%), Romania (67\%), and Taiwan (65\%), all reflecting the well-known wide geographic variation

Table 2: Clinical characteristics of newly diagnosed type 1 adults who developed DKA from September 2017 to May 2019 G.C in DURH.

\begin{tabular}{|c|c|c|c|}
\hline \multicolumn{2}{|l|}{ Variable } & \multirow{2}{*}{$\begin{array}{l}\text { Frequency } \\
161 \\
260\end{array}$} & \multirow{2}{*}{$\begin{array}{l}\text { Percent } \\
38.0 \\
62.0\end{array}$} \\
\hline First degree relatives & $\begin{array}{l}\text { Yes } \\
\text { No }\end{array}$ & & \\
\hline Do you present with DKA & $\begin{array}{l}\text { Yes } \\
\text { No }\end{array}$ & $\begin{array}{l}160 \\
261\end{array}$ & $\begin{array}{l}38.0 \\
62.0\end{array}$ \\
\hline Knowledge about DKA/DM & $\begin{array}{l}\text { Yes } \\
\text { No }\end{array}$ & $\begin{array}{l}202 \\
219\end{array}$ & $\begin{array}{l}48.0 \\
52.0\end{array}$ \\
\hline $\begin{array}{l}\text { Do you have Sign and symptoms of DKA/DM } \\
\text { before the onset of DKA }\end{array}$ & $\begin{array}{l}\text { Yes } \\
\text { NO }\end{array}$ & $\begin{array}{l}421 \\
0\end{array}$ & $\begin{array}{l}100.0 \\
0.0\end{array}$ \\
\hline Which sign and symptoms of DKA/DM & $\begin{array}{l}\text { Polydepsia } \\
\text { Polyuria and polydepsia } \\
\text { Polydepsia and weight lost } \\
\text { Polyuria and weight lost }\end{array}$ & $\begin{array}{l}7 \\
265 \\
56 \\
93\end{array}$ & $\begin{array}{l}1.7 \\
62.9 \\
13.3 \\
22.1\end{array}$ \\
\hline Infection before onset of DKA & $\begin{array}{l}\text { Yes } \\
\text { No }\end{array}$ & $\begin{array}{l}168 \\
253\end{array}$ & $\begin{array}{l}39.9 \\
60.1\end{array}$ \\
\hline Which infections & $\begin{array}{l}\text { Headache } \\
\text { Fever } \\
\text { Cough } \\
\text { Urinary compliant }\end{array}$ & $\begin{array}{l}81 \\
38 \\
89 \\
92\end{array}$ & $\begin{array}{l}19.2 \\
9.0 \\
21.1 \\
21.9\end{array}$ \\
\hline
\end{tabular}


Citation: Eskeziya A, Girma Z, Mandefreo B, et al. (2020) Prevalence of Diabetic Keto Acidosis and Associated Factors among Newly Diagnosed Patients with Type One Diabetic Mellitus at Dilla University Referral Hospital, September 9th/2017 - May 30th/2019: South Ethiopia; Crossectional Study. J Healthcare 3(1):33-38

Table 3: Bivariate analysis and multivariate analysis in newly diagnosed type 1 adults in DURH September $9^{\text {th }} / 2017-$ May30 th $/ 2019$.

\begin{tabular}{|c|c|c|c|c|c|c|}
\hline \multicolumn{2}{|l|}{ Variables } & \multicolumn{2}{|l|}{ DKA } & \multirow{3}{*}{$\begin{array}{l}\text { COR } \\
1.799(1.544-2.097) \\
\end{array}$} & \multirow{3}{*}{\begin{tabular}{|l} 
AOR \\
$3(2-10)$ \\
\end{tabular}} & \multirow{3}{*}{\begin{tabular}{|l|} 
P Value \\
$0.005^{*}$ \\
\end{tabular}} \\
\hline & & \multirow{2}{*}{$\begin{array}{l}\text { Yes } \\
154(54.0)\end{array}$} & \multirow{2}{*}{$\begin{array}{l}\text { No } \\
131(46.0)\end{array}$} & & & \\
\hline Age & $18-25$ & & & & & \\
\hline & $25-35$ & $6(4.4 \%)$ & $130(95.6)$ & 1.00 & 1.00 & \\
\hline \multirow{2}{*}{$\begin{array}{l}\text { Sign and symptom of DKA } \\
\text { in the last } 2 \text { wks }\end{array}$} & Yes & $58(28.7)$ & $144(71.0)$ & $1.539(0.537-4.410)$ & $0.825(0.001-1.878)$ & $0.022^{*}$ \\
\hline & No & $102(46 \%)$ & $117(53.4 \%)$ & 1.00 & 1.00 & \\
\hline \multirow[t]{2}{*}{ First degree relative } & Yes & $158(98.0)$ & $3(1.9 \%)$ & $1.958(1.78-2.244)$ & $16(6.8-38)$ & $0.008^{*}$ \\
\hline & No & $2(0.8 \%)$ & $258(99.0)$ & 1.00 & 1.00 & \\
\hline \multirow{2}{*}{$\begin{array}{l}\text { History of infection prior } \\
\text { to DKA }\end{array}$} & Yes & $159(95.2 \%)$ & $8(4.8 \%)$ & $1.963(1.711-2.252)$ & $46(12-84)$ & $0.02^{*}$ \\
\hline & No & $1(0.4 \%)$ & 252 (99.0) & 1.00 & 1.00 & \\
\hline
\end{tabular}

${ }^{*}$ - indicates statistically significant results at $\mathrm{p}$ value $<0.05$.

COR: Crude Odds Ratio; AOR: Adjusted Odds Ratio; $\mathrm{Cl}$ : Confidence Interval; ${ }^{*} i m p l i e s:$ Only covariates that have strong association ( $\left.<<0.2\right)$ at the bivariate level were included in the multivariate binary logistic regression to control for confounding.

in frequency of DKA at onset of adult diabetes mellitus [8]. The differing in magnitude might be explained by differences in study population, the back-ground prevalence of diabetes in the given population, presence or absence of family history of T1DM, socioeconomic status, delayed diagnosis and treatment as well as the definition of DKA used in the particular study [9]. A study conducted in black lion hospital found the magnitude of DKA to be $80 \%$, when we compare with our study, it is high which might be due to the smaller sample size (143), the study was conducted only in black lion hospital and because severe cases are referred to black lion hospital the magnitude might be exaggerated [3].

Another retrospective study conducted in Benin teaching hospital found the frequency of DKA in newly diagnosed adults to be $77.1 \%$ [9], and this frequency is higher relatives to this study because this study included adults $b / n$ 15-35 years of age and because the study period was 15 years. In systematic review on 40 studies in 31 countries on the associated factors of DKA on newly diagnosed adults, age was the most common factor described: 32 studies reported the effect of age on presentation [1] and this is consistent with our research. In the systematic review that included 40 studies, three studies reported on the influence of parental education and of these studies the study in German did not find significant association on developing DKA [16], similar to this study our research did not find significant association. Three studies examined the effect of family income and two European studies found that family income had no significant effect on risk of presenting in diabetic ketoacidosis and this result is consistent with our results [17]. This might be due to the reason that even though parents who get higher income and having higher level of education they may not be aware of DM/DKA and seek medical advice earlier. Although having a first degree relative with type1 Diabetes decreased the frequency of diabetic ketoacidosis in three studies, [17] it did not predict a diagnosis of new onset diabetes before progression to diabetic ketoacidosis, this might be because in adults it is very difficult to find the classical symptoms of DKA which are polyuria, polydepsia and weight loss so the adults might not consider the other sign and symptoms of DKA as the sign of DKA.
In this cross-sectional study the odds of developing DKA in newly diagnosed DM Adults who know the sign and symptoms of DM have 1.01 times less likely to develop DKA at primary diagnosis of DM than who do not know the sign and symptoms of DM. This is because adults who know the sign and symptoms of DM might seek health care before they develop DKA. Three studies in the systematic review by Usher Smith JA, et al. included data on the effect of a preceding infection or febrile illness. In two, a history of infection or febrile illness was associated with an increased risk of diabetic ketoacidosis [17] and similar to this our study found the odds of Adults who have no sign and symptoms of infection 1-2 weeks before developing DKA are 1.5 times less likely to develop DKA than adults who do have the sign and symptoms of infection and $p$ value of 0.002 . Infection is known to cause inflammation, pro-inflammatory Cytokine release, and a counter regulatory response that collectively lead to insulin resistance and metabolic decomposition [17]. As strength of this study, this study includes both the clinical and socio economic factors secondary data and Gives an insight for researchers especially for prospective study. Some of the limitations of the study are: first, the study using secondary data have incomplete data.

\section{Conclusions and Recommendations}

The overall magnitude of DKA in adults with newly diagnosed type 1 diabetes is relatively high. In particular, adults between 15-25 years of age have a high risk of DKA at onset. Age, having first degree relatives with DM and infection prior to DKA are found to be the significant explanatory variable of DKA in primary onset of DM. Based on our finding we recommend the Diabetic Association to give different workshop for health professional and to work on awareness creation for the society using different communication media. Based on the finding of this study, we, researchers of this work recommend all the health facilities to give health education about the sign and symptoms of DM and the acute complications of DM, we recommend the health professionals to give attention on treating adults with infection that don't have known sign and symptoms of DKA. And lastly we recommend further 
Citation: Eskeziya A, Girma Z, Mandefreo B, et al. (2020) Prevalence of Diabetic Keto Acidosis and Associated Factors among Newly Diagnosed Patients with Type One Diabetic Mellitus at Dilla University Referral Hospital, September 9th/2017 - May 30th/2019: South Ethiopia; Crossectional Study. J Healthcare 3(1):33-38

prospective study to Health science Researchers on protective factors and on complications of DKA.

\section{Declarations}

\section{Ethics approval and consent of participants}

Before data collection ethical clearance per protocol unique number: 007/17-13 was secured from Institutional Review Board (IRB) of Dilla university on September $3^{\text {rd }} / 2017$. Permission letter was taken from medical director of Dilla university hospital. Verbal consent from each study subject was asked and secured after detail explanation about the main purpose of the study

\section{Consent for publication}

"Not applicable".

\section{Availability of data and materials}

"The data that support the findings of this study has a sort of identifier of individual participants and researcher reserved to send it".

\section{Competing of interest}

The author declares he has no conflict of interest.

\section{Funding}

"Not applicable".

\section{Author Contributions}

AH involved idea conception for proposal development, writing up of proposal. ZG \& BM also contributed in supervising of data collection and data quality assurance. AEA involved in writing up of results and discussion, and in writing manuscript. All authors read and approved the final manuscript.

\section{Acknowledgments}

The authors would like to thank Dilla University, Management bodies of DURH for their cooperation and support, Data collectors for their responsible data collection.

\section{References}

1. Farsani SF, Brodovicz K, Soleymanlou N, et al. (2017) Incidence and prevalence of diabetic ketoacidosis (DKA) among adults with type 1 diabetes mellitus (T1D): A systematic literature review. BMJ Open 7.

2. Worku D, Hamza L, Woldemichael K (2010) Patterns of diabetic complications at Jimma university specialized hospital, southwest ethiopia. Ethiopian Journal of Health Sciences 20: 33-39.
3. Lester FT, Abdulkadir J, Larson D, et al. (1976) Diabetes mellitus: Clinical features in 404 Ethiopians. Ethiopian Medical Journal 14: 185-198.

4. Hardern R, Quinn N (2003) Emergency management of diabetic ketoacidosis in adults. Emergency Medicine Journal 20: 210-213.

5. Qari FA (2003) Bacteremia and septicemia in diabetic patients in western saudi arabia. Saudi Medical Journal 24: 1064-1067.

6. Umpierrez GE, Kitabchi AE (2003) Diabetic ketoacidosis: Risk factors and management strategies. Treatments in Endocrinology 2: 95-108.

7. Moore SR, Rozycki GS (2004) Department of surgery, emory university school of medicine, atlanta, georgia. Archives of Surgery 139: 359-361.

8. Barski L, Nevzorov R, Lotkowitz A, et al. (2009) Thyrotoxic hypokalemic periodic paralysis as the presenting symptom in a young Ashkenazi Jewish man. The Israel Medical Association journal 11: 573-574.

9. Chijioke A, AdamuAM, Makusidi AM (2010) Mortality patterns among type 2 diabetes mellitus patients in llorin, Nigeria. Journal of Endocrinology, Metabolism and Diabetes of South Africa 15: 79-82.

10. Ogbera AO, Awobusuyi J, Unachukwu C, et al. (2009) Clinical features, predictive factors and outcome of hyperglycaemic emergencies in a developing country. BMC Endocrine Disorders 9: 9.

11. Snorgaard O, Eskildsen PC, Vadstrup S, et al. (1989) Diabetic ketoacidosis in Denmark: Epidemiology, incidence rates, precipitating factors and mortality rates. Journal of Internal Medicine 226: 223-228.

12. Otieno CF, Kayima JK, Omonge EO, et al. (2005) Diabetic ketoacidosis: Risk factors, mechanisms and management strategies in sub-Saharan Africa: Areview. East African Medical Journal 82: 197-203.

13. Gill G (1990) Practical management of diabetes in the tropics. Tropical Doctor 20: 4-10.

14. Lester FT (1980) Ketoacidosis in Ethiopian diabetics. Diabetologia 18: 375-377.

15. Wolfsdorf J, Glaser N, Sperling MA (2006) Diabetic ketoacidosis in infants, children, and adolescents: A consensus statement from the american diabetes association. Diabetes Care 29: 11501159.

16. Dunger DB, Sperling MA, Acerini CL, et al. (2004) European society for paediatric endocrinology/lawson wilkins pediatric endocrine society consensus statement on diabetic ketoacidosis in children and adolescents. Pediatrics 113: e133-e140.

17. Kitabchi AE (2005) Hyperglycemic crises: Improving prevention and management. American Family Physician 71: 1659-1660.

DOI: $10.36959 / 569 / 457$

Copyright: (c) 2020 Eskeziya A, et al. This is an open-access article distributed under the terms of the Creative Commons Attribution License, which permits unrestricted use, distribution, and reproduction in any medium, provided the original author and source are credited. 